ReVISTA de BIOLOGía TROPICAL

\title{
Poblaciones de tiburones en las bahías Chatham y Wafer del Parque Nacional Isla del Coco, Costa Rica
}

\author{
Ilena Zanella ${ }^{1}$, Andrés López-Garro ${ }^{1}$, Frank Martínez ${ }^{1}$, Geiner Golfín-Duarte ${ }^{2} \&$ Keylor Morales ${ }^{2}$ \\ 1. Asociación Conservacionista Misión Tiburón, Playas del Coco, Guanacaste, Costa Rica; alopez@misiontiburon.org; \\ izanella@misiontiburon.org, fmartinezl123@hotmail.com \\ 2. Parque Nacional Isla del Coco, Área Conservación Marina Isla del Coco, Sistema Nacional de Áreas de Conservación, \\ Costa Rica; geiner.golfin@sinac.go.cr; keylor.morales@sinac.go.cr
}

Recibido 04-III-2014. Corregido 06-IV-2015. Aceptado 02-VI-2015.

\begin{abstract}
Shark populations in Chatham and Wafer bays, Isla del Coco National Park, Costa Rica. At least fourteen species of sharks live in the Marine Protected Area of Isla del Coco National Park (ICNP), some are resident species such as the whitetip reef shark (Triaenodon obesus); others are migratory and visit the National Park throughout the year, e.g. the scalloped hammerhead shark (Sphyrna lewini) and the whale shark (Rhincodon typus). Between March 2010 and August 2012 six expeditions out to the ICNP were carried, during this period 25 night trips (from 18:00 to 22:00 hr) for shark tagging were conducted in the Wafer and Chatham bays. During those trips, 185 sharks were analyzed: $81.1 \%$ were whitetip reef sharks (T. obesus), $16.7 \%$ were blacktip sharks (Carcharhinus limbatus), $1.1 \%$ were blacktip reef sharks (Carcharhinus melanopterus) and $1.1 \%$ were silvertip sharks (Carcharhinus albimarginatus). The most common species in Bahía Chatham was T. obesus, while the most common one in Bahía Wafer was C. limbatus, which represented $80.9 \%$ and $80.5 \%$ of the total sharks caught, respectively. Regarding $T$. obesus, a sex ratio of 1.2 males per female was estimated $\left(\mathrm{X}^{2}=0.09 ; \mathrm{p}=0.17\right)$; in turn a sex ratio of 1.01 males per female was observed $\left(\mathrm{X}^{2}=0.04 ; \mathrm{p}=0.79\right)$ for $C$. limbatus. The average total length for T. obesus and C. limbatus was $103.66 \pm 13.91 \mathrm{~cm}$ and $76.58 \pm 14.56 \mathrm{~cm}$ respectively; suggesting that black tip sharks use those bays during immature stages. The results show an interspecific spatial segregation of the most common resident sharks at ICNP, apparently the clear waters and the coral cover of Chatham Bay are preferred by T. obesus; while the turbid waters of the Genio River mouth are preferred by $C$. limbatus. Rev. Biol. Trop. 64 (Suppl. 1): S177-S186. Epub 2016 February 01.
\end{abstract}

Keywords: Whitetip shark, Blacktip shark, species segregation, Isla del Coco National Park, Costa Rica.

El drástico aumento de la presión pesquera ha afectado las poblaciones de especies marinas, tanto costeras como oceánicas a nivel global (Myers, Baum, Shepered, Powers, \& Peterson, 2007; Myers, \& Worm, 2003). Se estima que los océanos del mundo han perdido más del $90 \%$ de los grandes depredadores (Myers, \& Worm, 2003), siendo los tiburones, por sus características biológicas, particularmente afectados. Por su posición ecotrófica de depredadores tope, los tiburones tienen una menor tasa reproductiva y de mortalidad natural con respecto a otros organismos marinos como los peces óseos, crustáceos y moluscos. Estas características hacen que los tiburones sean particularmente vulnerables a la sobreexplotación pesquera (Rowe, \& Hutchings, 2003). A pesar de esto, en las últimas dos décadas la pesquería dirigida al tiburón se ha intensificado (Kitchell, Essington, Boggs, Schindler, \& Walters, 2002; Abercrombie, Clarke, \& Mahmood, 2005).

Esto en gran parte debido a la creciente demanda de aletas de tiburón en mercados asiáticos de países como China y Taiwán (Walker, 1998; Kitchell et al., 2002; Abercrombie et al., 2005). Por lo anterior, se han realizado esfuerzos para promover la conservación de los tiburones a nivel internacional. Por ejemplo, en el marco del Paisaje Marino del Pacífico Tropical 
Este (ETPS, por sus siglas en inglés) se han desarrollado proyectos para promover la conservación y manejo de los tiburones en México, Panamá, Costa Rica, Colombia y Ecuador (Hearn, Klimley, \& Peñaherrera, 2010a).

Investigadores de la región han estado colaborando en el estudio del comportamiento espacial y movimientos interinsulares, probando la conexión biológica de las poblaciones de tiburón martillo (Sphyrna lewini) entre las islas oceánicas de Malpelo, Galápagos e Isla del Coco (Hearn et al., 2010a, Hearn, Utreras, \& Henderson, 2010b; Bessudo et al. 2011). El Parque Nacional Isla del Coco (PNIC) se destaca en la región como uno de los sitios más importantes en cuanto a riqueza y abundancia de tiburones (Hearn et al., 2010a, b; Nalesso 2014).

En las aguas del PNIC se han reportado catorce especies de tiburones (Bussing, \& López, 2005; Cortés et al., 2012), algunas especies son residentes, como el tiburón punta blanca de arrecife (Triaenodon obesus), mientras que otras son altamente migratorias, como el tiburón martillo (S. lewini) y el tiburón ballena (Rhincodon typus). Las montañas submarinas, los islotes, y las bahías del PNIC representan hábitats esenciales (áreas de alimentación, descanso, limpieza, cópula) para las distintas especies de tiburones (ACMIC, 2002).

En las bahías localizadas en el norte del PNIC, Bahía Chatham y Bahía Wafer, se destaca la presencia del tiburón punta blanca de arrecife ( $T$. obesus) y el tiburón punta negra (Carcharhinus limbatus). Estudios previos (conteos subacuáticos) en Bahía Chatham estimaron una abundancia relativa para $T$. obesus de 49.5 \pm 10.4 tiburones/hr (Zanella, López, Golfín, \& Sáenz, 2012). Además, para esta especie los autores analizaron los estados reproductivos de los machos que habitan en Bahía Chatham, reportando la presencia de tiburones juveniles, sub-adultos y adultos, con un mayor abundancia de sub-adultos (Zanella et al., 2012).

Generalmente las bahías, al presentar aguas someras y protegidas, son utilizadas como áreas de crianza por los tiburones, brindando a los juveniles protección contra los depredadores y disponibilidad de alimento (Castro, 1993). Las hembras eligen estas áreas con el fin de disminuir la mortalidad natural de sus crías (Simpfendorfer, \& Milward, 1993; Merson, \& Pratt, 2001), ya que el éxito de una población con baja tasa reproductiva (como los tiburones) se basa en la supervivencia de sus juveniles (Simpfendorfer, \& Milward, 1993). A pesar de la importancia que tienen las bahías del PNIC para los tiburones, pocas investigaciones científicas se han desarrollado con el fin de estudiar las poblaciones de tiburones presentes en las bahías Chatham y Wafer. Debido a esto, el objetivo de este manuscrito fue caracterizar las especies y poblaciones de tiburones más comunes que habitan esas dos bahías del PNIC.

\section{MATERIALES Y MÉTODOS}

Área de estudio: La Isla del Coco se ubica en aguas oceánicas del Pacífico costarricense $\left(5^{\circ} 32^{\prime} \mathrm{N}-87^{\circ} 04^{\prime} \mathrm{W}\right)$ aproximadamente a $500 \mathrm{~km}$ de la provincia de Puntarenas. Declarado como Parque Nacional en 1978 e inscrito como Sitio Patrimonio de la Humanidad en 1997, la Isla del Coco posee hábitats críticos para diferentes especies pelágicas, particularmente para tiburones (Cortés, 2008; Friedlander et al., 2012). Tal es el caso de la Bahía Chatham y Bahía Wafer, la primera se ubica entre las coordenadas $5^{\circ} 33^{\prime} 25.28^{\prime \prime} \mathrm{N}-87^{\circ} 02^{\prime} 53.11^{\prime \prime} \mathrm{W}$ y $5^{\circ} 33^{\prime} 04.71^{\prime \prime} \mathrm{N}-87^{\circ} 02^{\prime} 13.76^{\prime \prime} \mathrm{W}$ y tiene una profundidad de $10-20 \mathrm{~m}$. Se caracteriza por la presencia de aguas cristalinas (con una menor cantidad de nutrientes y sedimentos) y distintas acumulaciones de rocas y arrecifes coralinos. Bahía Wafer se ubica entre las coordenadas $5^{\circ} 32^{\prime} 56.33^{\prime \prime} \mathrm{N}-87^{\circ} 03^{\prime} 44.96^{\prime \prime} \mathrm{W}$ y $5^{\circ} 32^{\prime} 47.35^{\prime}$ " $\mathrm{N}-8^{\circ} 03^{\prime} 27.48^{\prime}$ " W y se caracteriza por ser una bahía poco profunda o somera, entre los 10-20 m, con la presencia de bancos rocosos y canales arenosos. En la Bahía Wafer desemboca el Río Genio, río de mayor tamaño y con la cuenca de mayor captación de la Isla, por esta razón las aguas de la bahía presentan turbidez y gran cantidad de sedimentos (Sinergia 69, 2002; Sibaja-Cordero, \& Cortés, 
2010; Sibaja-Cordero, Troncoso, Benavides, \& Cortés, 2012).

Captura de tiburones: Se realizaron seis expediciones al PNIC para marcar tiburones como parte del estudio "Dinámica poblacional del tiburón punta blanca de arrecife (Triaenodon obesus) en las bahías del PNIC": 1) Diciembre 2010, 2) Marzo 2011, 3) Marzo 2012, 4) Mayo 2012, 5) Agosto 2012 y 6) Diciembre 2012. De esta manera los tiburones analizados fueron capturados durante recorridos en Bahía Chatham, Bahía Wafer y zonas aledañas (Isla Manuelita e Isla Ulloa), entre las 17:00-22:00 hr. Se utilizó una cuerda de mano con anzuelos circulares \#5 modificados ( $\sin$ muesca para minimizar el daño físico en el tiburón) con carnada de calamar gigante y calamar torpedo (Loliginidae). Con el fin de relacionar la abundancia (número de tiburones) de las diferentes especies de tiburones con las bahías y zonas aledañas del PNIC, se realizó un análisis de correspondencia (AC) con el programa InfoStat (Di Rienzo et al., 2008). Por último, para monitorear las poblaciones de tiburones que habitan las bahías y zonas aledañas al PNIC, se estimó la abundancia relativa como CPUE (Captura por Unidad de Esfuerzo), a partir del número total de tiburones capturados dividido por las horas que tomó capturarlos. Para el análisis de CPUE se utilizaron solo los tiburones punta blanca (T. obesus) capturados en Bahía Chatham.

Datos biométricos: Los tiburones capturados fueron subidos a la embarcación y se les retiró el anzuelo. Inmediatamente, se tomó la posición geográfica con un GPS, la hora de captura, profundidad de captura y temperatura superficial del agua. A cada tiburón se le evaluó el sexo (mediante la presencia o ausencia de los hemipenes) y fue medido con un ictiómetro: longitud total (LT: medida desde la punta del hocico, hasta el extremo final de la aleta caudal, con un error de medición de $\pm 0.1 \mathrm{~cm}$ ). A los tiburones machos se les determinó el estado reproductivo según la calcificación de los hemipenes, utilizando la clasificación para
Mustelus schmitti de la Patagonia (Chiaramonte, \& Pettovello, 2000), la cual se describe a continuación:

- $\quad$ Estado I - Hemipenes cortos y suaves.

- Estado II - Hemipenes cortos y parcialmente calcificados.

- $\quad$ Estado III - Hemipenes totalmente calcificados, la base de los hemipenes puede ser rotada $180^{\circ}$ y vuelve a su posición original. Presencia de semen en los hemipenes.

Para las especies de tiburones más capturados se calcularon las proporciones sexuales, y se utilizó la prueba Chi-cuadrado (Programa InFostat) para evaluar diferencias en las proporciones de machos y hembras. Los valores de longitud total media de las especies de tiburones más capturados fueron estimados uniendo los datos provenientes de Bahía Chatham, Bahía Wafer y zonas aledañas. Además, se calculó el mínimo (min), máximo (max) y la desviación estándar (DE) de las longitudes para las especies de tiburones más capturados.

\section{RESULTADOS}

Captura de tiburones: Se realizaron 25 recorridos nocturnos, entre las 18:00-22:00 hr. De estos, el $56 \%$ fueron en Bahía Chatham

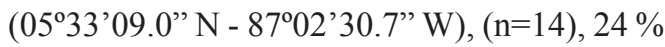
en Bahía Wafer $\left(05^{\circ} 32^{\prime} 42.2^{\prime \prime} \mathrm{N}-87^{\circ} 03^{\prime} 45.3\right.$ " W) $(n=6), 12 \%$ en Ulloa $\left(05^{\circ} 32 ' 58.4 " \mathrm{~N}\right.$ $\left.87^{\circ} 01^{\prime} 55.6^{\prime \prime} \mathrm{W}\right)(\mathrm{n}=3)$ y $8 \%$ en Manuelita $\left(05^{\circ} 33^{\prime} 42.7^{\prime \prime} \mathrm{N}-87^{\circ} 02^{\prime} 48.9^{\prime \prime} \mathrm{W}\right)(\mathrm{n}=2)$. En los sitios de marcaje la profundidad y temperatura superficial del agua promedio fue de $12.1 \pm 2.5 \mathrm{~m}($ mínimo=7.5 $\mathrm{m}$ y máximo $=19.1 \mathrm{~m})$ y $28.4 \pm 1.2^{\circ} \mathrm{C}$, respectivamente.

Durante los 25 recorridos se capturaron y analizaron 185 tiburones. De los cuales el 81.1 $\%$ fueron tiburones punta blanca de arrecife ( $T$. obesus, $\mathrm{n}=150$ ), $16.7 \%$ tiburones punta negra (C. limbatus, $\mathrm{n}=31$ ), $1.1 \%$ tiburones punta negra de arrecife (Carcharhinus melanopterus, $\mathrm{n}=2$ ) y $1.1 \%$ tiburones punta plateada (Carcharhinus albimarginatus, $\mathrm{n}=2$ ). Con respecto al total de tiburones analizados en cada zona 
de muestreo, el $70.27 \%$ fueron capturados en Bahía Chatham $(\mathrm{n}=130), 20.55 \%$ en Bahía Wafer ( $\mathrm{n}=38), 4.86 \%$ en Ulloa $(\mathrm{n}=9)$ y $4.32 \%$ en Manuelita $(\mathrm{n}=8)$. A su vez más del $80.9 \%$ y $80.5 \%$ de los tiburones $T$. obesus y C. limbatus se capturaron en Bahía Chatham y Bahía Wafer, respectivamente.

En cuanto al análisis de correspondencia (AC), los dos primeros ejes explican el 96.6 $\%$ de la variación de la relación entre especies de tiburones y las áreas (biplot) (Fig. 1). En este análisis la distancia entre las especies y las áreas refleja su grado de dependencia: entre más cerca se encuentren, más fuerte será la relación. Además, la distancia perpendicular de las especies y las áreas con respecto al primer eje (x) y segundo eje (y) representa la magnitud del coeficiente de regresión de las distintas relaciones (Fig. 1). En el análisis CA, se observa que existe una fuerte relación entre el tiburón punta blanca de arrecife (T. obesus) y la Bahía Chatham, mientras que el tiburón

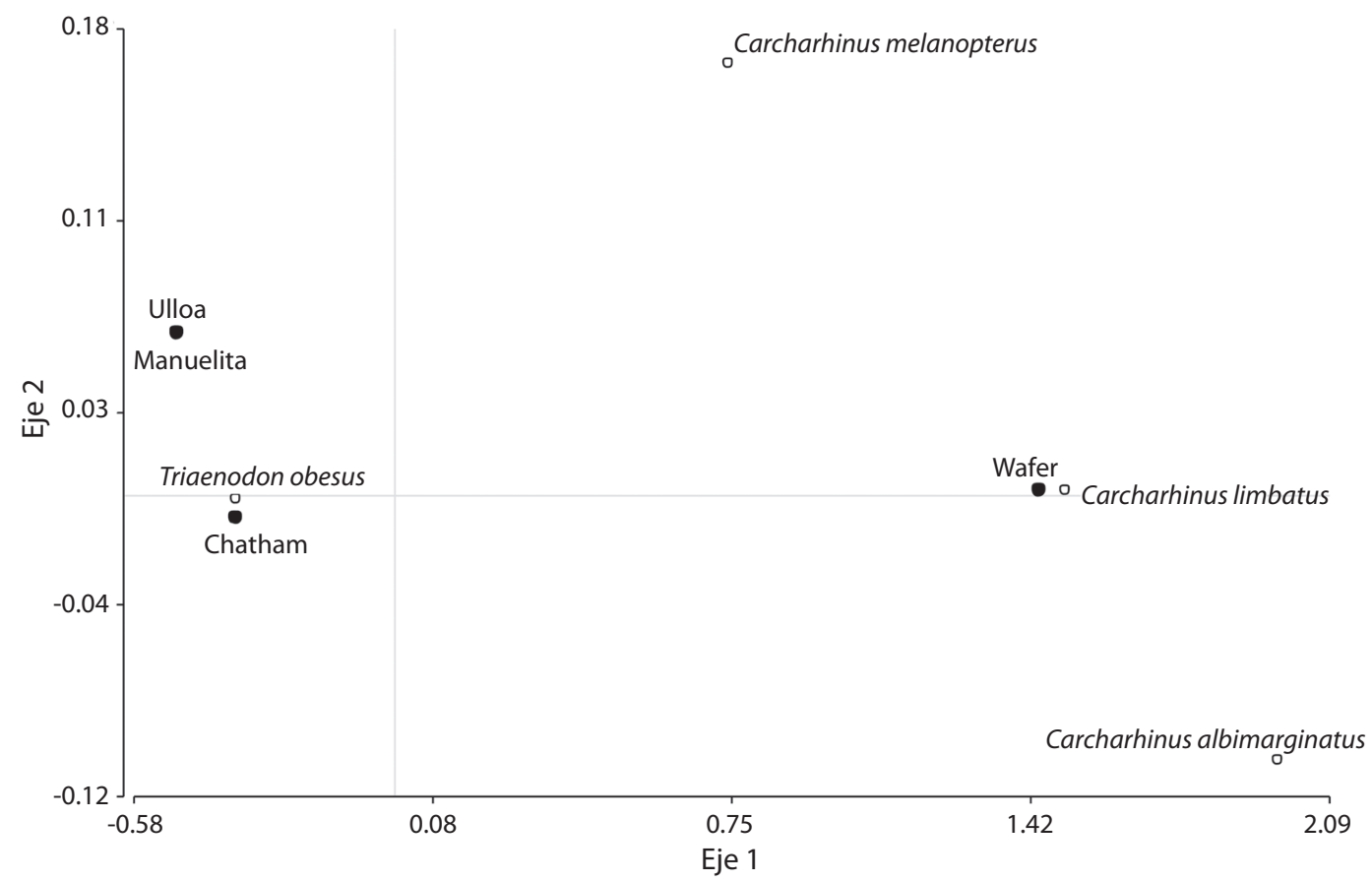

Fig 1. Análisis de correspondencia (CA) entre especies de tiburones y las bahías y zonas aledañas del PNIC. Costa Rica, 2010-2013.

Fig. 1. Correspondence analysis between sharks species and the bays and adjacent areas of ICNP. Costa Rica, 2010-2013. punta negra $(C$. limbatus $)$ se relaciona más con la Bahía Wafer (Fig. 1) (Di Rienzo et al., 2008).

Los análisis de las capturas durante los recorridos, revelan que el $39.2 \%$ de los organismos analizados fueron capturados entre las 19:00 y 20:00 hr. A su vez, el $32.8 \%$ de los tiburones se capturaron entre las 20:00 y 21:00hr, mientras que solo el $6.4 \%$ fueron capturados entre las 17:00 y 18:00 hr (Fig. 2). punta blanca estimada para las Bahías Chatham fue en promedio de $6.04 \pm 13.37$ tiburones por hora, con un mínimo y máximo de 1.97 y 12.00 tiburones por hora respectivamente.

Para la especie más abundante el tiburón $T$. obesus, el $45.9 \%$ fueron hembras y el $54.1 \%$ machos ( $\mathrm{n}=127)$, para una proporción sexual de 1.2 machos por cada hembra, la diferencia entre proporciones no fue significativa $\left(\chi^{2}=0.09\right.$, $\mathrm{p}=0.17$ ). La mayoría de los machos analizados $(50.7 \%)$ se encontraban en el estado II (subadultos); mientras que la menor proporción de

¿Carcharhinus melanopterus La CPUE o abundancia relativa de tiburones 


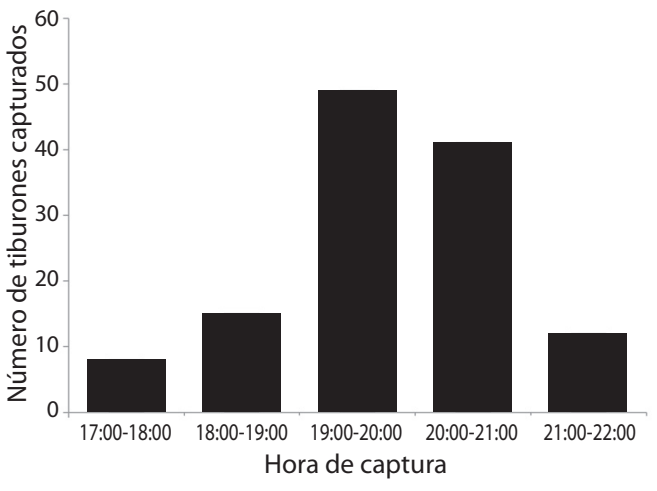

Fig. 2. Número de tiburones punta blanca (T. obesus) capturados entre las 17:00 y 22:00 en las bahías Chatham y Wafer y zonas aledañas del PNIC. Costa Rica, 2010-2013 $(\mathrm{n}=125)$.

Fig. 2. Number of sharks caught between the 17:00 and 22:00 at Chatham and Wafer bays, and adjacent areas in the ICNP. Costa Rica 2010-2013 ( $\mathrm{n}=125)$.

los machos correspondió al estado I, inmaduros (crías y juveniles) (21.3\%) (Fig. 3). La longitud total promedio de los tiburones T. obesus analizados fue $103.66 \pm 13.91 \mathrm{~cm}$, con una longitud total máxima de $146.0 \mathrm{~cm}$ y una mínima de $64.0 \mathrm{~cm}$. Con respecto a la distribución de los tiburones $T$. obesus analizados, más del $70 \%$ de los organismos registraron longitudes totales entre 90 y $120 \mathrm{~cm}$. Mientras solo cerca del $10 \%$ presentaron longitudes mayores a los $120 \mathrm{~cm}$ (Fig. 4).

A su vez, la longitud promedio del tiburón punta negra (C. limbatus) fue de $76.58 \pm 14.56 \mathrm{~cm}$, con una longitud total máxima de $123.0 \mathrm{~cm}$ y una mínima de $52.0 \mathrm{~cm}$. Con respecto a la distribución de longitudes de los tiburones $C$. limbatus analizados, cerca del $26.6 \%$ y $63.6 \%$ de los organismos registraron longitudes totales entre los $50-65 \mathrm{~cm}$ y $65-95 \mathrm{~cm}$, respectivamente (Fig. 5). Por último para $C$. limbatus se obtuvo una proporción sexual 1.01:1 machos por cada hembra $\left(\chi^{2}=0.04, p=0.79\right)$.

\section{DISCUSIÓN}

El presente estudio revela que en las Bahías Chatham, Wafer y zonas aledañas habitan al menos cuatro especies de tiburones, todos pertenecientes a la familia Carcharhinidae. Al

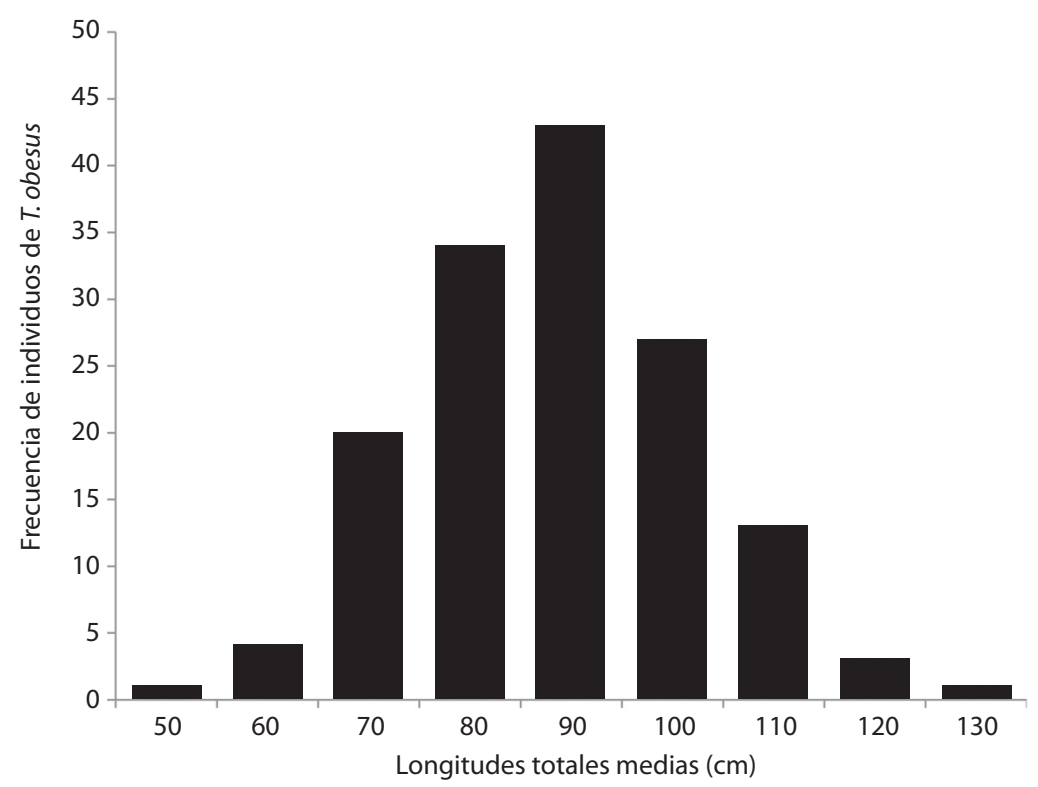

Fig. 3. Distribución de frecuencia por Longitud Total (LT) de los tiburones punta blanca analizados en las bahías Chatham y Wafer y zonas aledañas del PNIC ( $n=147)$. Costa Rica, 2010-2013.

Fig. 3. Frequency distribution of total length for the white tip reef shark $(n=147)$ analyzed at Chatham and Wafer bays, and adjacent areas in the ICNP. Costa Rica 2010-2013. 


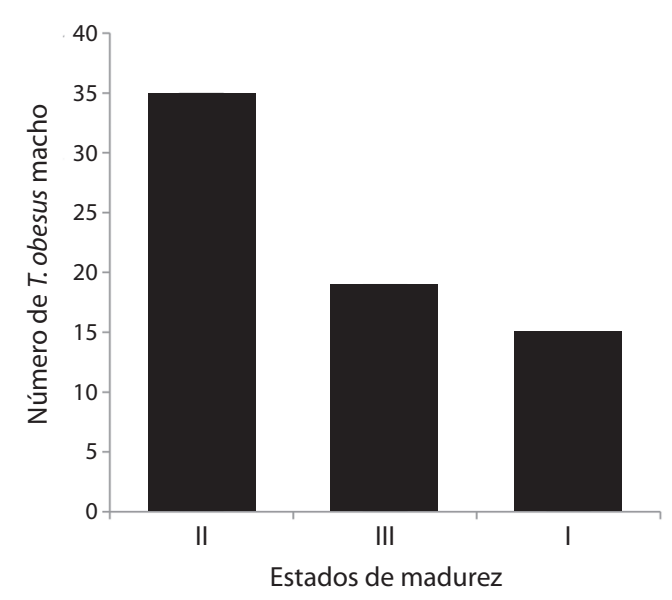

Fig. 4. Distribución de frecuencia por estados de madurez de los tiburones punta blanca $(n=36)$ machos analizados en las bahías Chatham y Wafer y zonas aledañas del PNIC. Costa Rica, 2010-2013.

Fig. 4. Frequency distribution of sexual maturity stage for males of whitetip reef shark $(n=36)$ analyzed at Chatham and Wafer bays, and adjacent areas in the ICNP. Costa Rica 2010-2013.

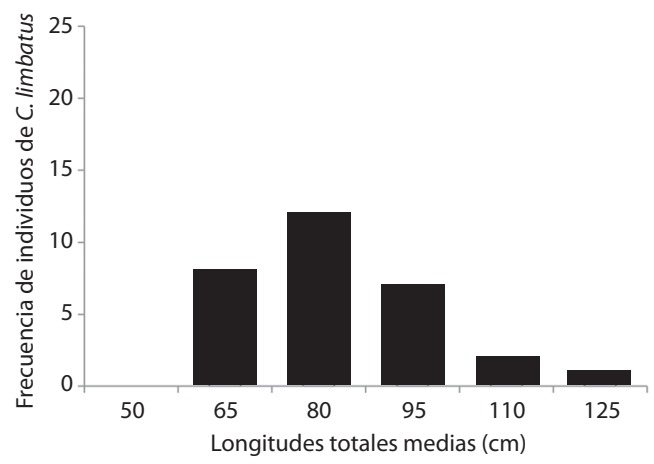

Fig. 5. Distribución de frecuencia por Longitud Total (LT) de los tiburones punta negra analizados $(n=31)$ en las bahías Chatham y Wafer y zonas aledañas del PNIC. Costa Rica, 2010-2013.

Fig. 5. Frequency distribution of total length for black tip sharks $(n=31)$ analyzed at Chatham and Wafer bays, and adjacent areas in the ICNP. Costa Rica 2010-2013.

igual que en el PNIC, en otras zonas marinas de aguas tropicales, dicha familia es muy abundante y domina tanto en las capturas pesqueras, como en los censos submarinos. Por ejemplo, en la Reserva Marina de las Galápagos, cuatro de las siete especies capturadas ilegalmente en el Área Marina Protegida pertenecen a la familia Carcharhinidae (Carr et al., 2013). En La Gran Barrera de Coral en Australia, los análisis a bordo de embarcaciones pesqueras artesanales revelan que de las 26 especies de tiburones capturados por los trasmallos, cerca del $80 \%$ pertenecen a la familia Carcharhinidae (Alastair et al., 2011). A su vez, Tavares (2005) en el archipiélago de Los Roques en Venezuela analizó 15 diferentes especies provenientes de las pesquerías de palangre, de las cuales más del $60 \%$ fueron clasificados dentro de la familia Carcharhinidae. De la misma forma para el Refugio Nacional de Vida Silvestre de Estados Unidos "Atolón-Palmira" ubicado en el Pacífico Central, las tres especies de tiburones más comunes avistadas en conteos subacuáticos pertenecen a la familia Carcharhinidae, a saber, Carcharhinus amblyrhynchos, C. melanopterus y T. obesus (McCauley, Mclean, Bauer, Young, \& Micheli, 2012).

Los análisis de las capturas revelan que más del $70 \%$ de los tiburones fueron capturados en las bahías del PNIC entre las 19:00 y las 21:00 hr. Esto nos hace suponer que durante las primeras horas de la noche los tiburones se vuelven más activos y se dedican a la alimentación, ya que este comportamiento ha sido reportado para la especie más abundante en las bahías del PNIC, el tiburón T. obesus. Esta especie suele ser avistada descansando durante el día sobre el fondo marino cerca de arrecifes coralinos y rocosos, mientras en horas de la noche se vuelve muy activo (Randall, 1977). Incluso esta especie manifiesta niveles más altos de actividad metabólica durante la noche que en el día (Whitney, Papastamatiou, Holland, \& Lowe, 2007; Fitzpatrick, Abrantes, Seymour, \& Barnett, 2011). Como se mencionó antes, el tiburón $T$. obesus fue la especie más abundante en las bahías del PNIC, esto explica que la mayoría de las capturas totales se efectuaran entre las 19:00 y 21:00 hr.

Por otro lado más del $80 \%$ de los tiburones punta blanca se registraron en la Bahía Chatham. De la misma forma el AC demostró una fuerte relación entre $T$. obesus y dicha bahía. Por lo tanto se sugiere una preferencia 
de esta especie por las aguas someras, con poco sedimento y con abundante cobertura coralina presentes en la bahía Chatham. Según Randall (1977), el tiburón T. obesus usualmente es encontrado en aguas muy claras entre $\operatorname{los} 8$ y $40 \mathrm{~m}$ de profundidad en Madagascar. Además, Nelson y Johnson (1980) reportan como $T$. obesus es más abundante en arrecifes coralinos entre los 10 y $30 \mathrm{~m}$. De esta manera T. obesus sobresale por su abundancia en las bahías del PNIC, siendo el único tiburón de la familia Carcharhinidae que es bentónico y costero, dos factores asociados con una limitada dispersión y con un rango de distribución pequeño (Musick, Harbin, \& Compagno, 2004). Sumado a lo anterior T. obesus presenta un alto nivel de fidelidad, comparable a la que presentan muchos peces coralinos óseos, los pocos estudios referentes a sus movimientos, indican rangos diarios de distribución para $T$. obesus de alrededor $1 \mathrm{~km}^{2}$ con una dispersión máxima de 9-24 km después de varios años (Nelson, \& Johnson, 1980).

Sus hábitos residentes, convierten al tiburón $T$. obesus en una especie ideal para estudios poblaciones. A su vez, la alta abundancia reportada previamente para Bahía Chatham y alrededores del PNIC (Zanella et al., 2012), convierten a la población de $T$. obesus que habita en el PNIC en una de las más prístinas del mundo, donde la mortalidad por pesca es nula y por ende, la principal causa de mortalidad es natural, probablemente la depredación de otros tiburones de mayor tamaño que habitan las bahías como tiburón tigre (Galeocerdo cuvier) y el tiburón galápagos (Carcharhinus galapagensis) (López, Zanella, Martínez, Golfín, \& Pérez, 2016).

El ámbito de longitudes de los especímenes de T. obesus analizados (64-146 cm), concuerda con lo encontrado por Robbins (2006) en las capturas pesqueras comerciales en la Gran Barrera de Coral de Australia, donde los autores reportaron tallas de capturas de esta especie, entre los 70.0 y $140.0 \mathrm{~cm}$. A su vez, los análisis del estado reproductivo en machos del tiburón $T$. obesus, revelaron tiburones inmaduros (crías y sub-adultos) y maduros (adultos), lo cual confirma que esta especie no posee una segregación ontogénica espacial bien definida, en las bahías del PNIC, como sí se ha reportado para otras especies como el tiburón martillo $(S$. lewini) (Klimley, 1987; Torres, 2004; Zanella, López, \& Arauz, 2009), el tiburón toro (Carcharhinus leucas) y el tiburón punta negra $(C$. limbatus), especies en las cuales juveniles y adultos no conviven en los mismos hábitats (Heupel, \& Simpfendorfer, 2008; McCord, \& Lamberth, 2009). Cabe resaltar que, a pesar de que varios autores han descrito la selectividad de los artes de pesca (tamaño del anzuelo) sobre determinadas tallas de los tiburones (Afonso et al., 2011; Hanna et al., 2013), el amplio ámbito de longitudes registrada sugiere que el arte de pesca utilizado no afectó la probabilidad de captura de ciertas tallas de $T$. obesus en las bahías.

Con respecto a las otras especies de tiburones analizadas en las bahías, el tiburón $C$. limbatus fue la segunda especie más abundante, mostrando una significativa relación con Bahía Wafer. Los tiburones $C$. limbatus analizados en las bahías del PNIC presentaron una longitud total media cercana a los $75 \mathrm{~cm}$, por lo que todos los individuos fueron juveniles. Según Gaitán y López (2008), esta especie alcanza la madurez sexual entre los 120 y $190 \mathrm{~cm}$ en las hembras, y entre 135 y $180 \mathrm{~cm}$ en los machos. A su vez, Branstetter (1987) reportó para el Atlántico Oeste una longitud total de madurez para $C$ limbatus entre los 130-150 cm de longitud total, mientras que Tavares (2008) en Los Roques, Venezuela reporta las menores longitudes total de adultos de individuos de $C$. limbatus $152 \mathrm{~cm}$ hasta los $161 \mathrm{~cm}$. Para $C$. limbatus diferentes autores han reportado la utilización de bahías y bocas de ríos, donde incluso se han reportado para los juveniles algunas áreas de crianza, tal es el caso de Tavares (2008) quien reporta una para el Archipiélago Los Roques en Venezuela.

Conforme van creciendo los tiburones punta negra salen esporádicamente del área de crianza e incursionan en aguas más profundas (Heupel, Simpfendorfer, \& Hueter, 2004). Debido a esto, es probable que en el PN Isla del Coco los tiburones punta negra conforme van 
creciendo se trasladen hacia aguas profundas y con mayor influencia de corrientes oceánicas, esto debido a que algunos individuos adultos y preadultos son avistamos por buceos scuba en islotes como Roca Sucia ubicados a más de $1 \mathrm{~km}$ de la bahía Wafer.

Al igual que en otras zonas como en Los Roques y en el Atlántico Norte, en Bahía Wafer se analizó una misma proporción de hembras y machos del tiburón C. limbatus (Tavares, 2008; Branstetter, 1987). Por esta razón, se sugiere que las aguas turbias y arenosas de la bahía brindan alimentación y protección a las crías y juveniles de esta especie. Sin embargo, para poder afirmar que la Bahía Wafer es un área de crianza para el tiburón $C$. limbatus será necesario monitorear la población a mediano plazo. Estudios de marcaje acústico de esta especie en el Golfo de México han permitido comprobar como su rango de hábitat durante los primeros 6 meses de vida es muy restringido (Promedio $1 \mathrm{~km}^{2}$ al día) (Heupel et al., 2004). Esto refuerza la hipótesis de que bahía Wafer es un área de crianza, donde problamente los C. limbatus recién nacidos pasan la mayoría del día, de tal manera que conforme estos van creciendo, van realizando pequeñas salidas del área de crianza, hasta que la dejan permanente.

Otras especies como el tiburón C. albimarginatus y $C$. melanopterus fueron capturadas muy ocasionalmente en las bahías, ya que solo se analizaron dos individuos de cada una. Cabe resaltar que los individuos eran juveniles, por esta razón las bahías podrían estar siendo utilizadas como área de crianza por otras especies de tiburones menos abundantes. Sin embargo, será necesario comprobar este y otros supuestos, ya que la captura de dos individuos en diferentes momentos de C. melanopterus (especie recientemente reportada para el PNIC) nos hace suponer que en las aguas del PNIC habita una pequeña población de esta especie, un tiburón muy común del Indo-pacífico que, sin embargo fue hallado por primera vez en Bahía Chatham en el año 2012 (López, Zanella, Golfín, \& Pérez, 2012).

Finalmente, con el fin de confirmar el uso de hábitat de los tiburones en Bahía Chatham y Bahía Wafer se recomienda seguir monitoreando estas poblaciones con metodologías más avanzadas (telemetría acústica) que podrían brindar información más robusta para el manejo y conservación de las especies.

\section{AGRADECIMIENTOS}

Agradecemos al Área de Conservación Marina Isla del Coco y a los funcionarios del Parque Nacional Isla del Coco por habernos apoyado durante las expediciones con recursos, personal y embarcaciones. A la vez, la investigación fue posible gracias a los fondos aportados por Flying Sharks y Fundación Amigos de la Isla del Coco (FAICO).

\section{RESUMEN}

En el Parque Nacional Isla del Coco (PNIC) habitan al menos catorce especies de tiburones, algunas especies son residentes como el tiburón punta blanca de arrecife (Triaenodon obesus), mientras otras son migratorias. Tal es el caso de les tiburones martillo (Sphyrna lewini) quienes son avistados en el PNIC en diferentes meses del año. Entre marzo del 2010 y agosto del 2012 se realizaron seis expediciones al PNIC, durante las cuales se hicieron 25 recorridos nocturnos (entre las 18:00-22:00 hr) para el marcaje de tiburones. Durante los recorridos se analizaron 185 tiburones, de los cuales el $81.1 \%$ fueron tiburones punta blanca de arrecife ( $T$. obesus), $16.7 \%$ tiburones punta negra (Carcharhinus limbatus), $1.1 \%$ tiburones punta negra de arrecife (Carcharhinus melanopterus) y $1.1 \%$ tiburones punta plateada (Carcharhinus albimarginatus). A su vez más el $80.9 \%$ y $80.5 \%$ de los tiburones $T$. obesus y $C$. limbatus se capturaron en Bahía Chatham y Bahía Wafer, respectivamente. Las capturas en los recorridos revelan que el 39.2\% de los organismos se analizaron entre las 19:00 y 20:00 hr. Los resultados muestran una segregación espacial interespecífica de las especies más comunes en el PNIC. Se sugiere que las aguas cristalinas y con cobertura coralinas presentes en Bahía Chatham son preferidas por T. obesus, mientras que la desembocadura del Río Genio y las aguas turbias de Bahía Wafer son preferidas por C. limbatus.

Palabras claves: Tiburón punta blanca, tiburón punta negra, segregación de especies,Parque Nacional Isla del Coco, Costa Rica.

\section{REFERENCIAS}

Abercrombie, D. L., Clarke, S. C., \& Mahmood, S. S. (2005). Global-scale genetic identification of hammerhead sharks: Application to assessment of the 
international fin trade and law enforcement. Conservation Genetic, 6, 775-788.

ACMIC (2002). La presentación de informes periódicos sobre la aplicación de la convención del patrimonio mundial. Sección II: Estado de conservación de bienes específicos del Patrimonio Mundial. San José, Costa Rica: UNESCO.

Afonso, A., Hazin, F., Carvalho, F., Pacheco, J., Hazin, H., Kerstetter, D., Murie, D., \& Burgess, G. (2011) Fishing gear modifications to reduce elasmobranch mortality in pelagic and bottom longline fisheries off Northeast Brazil. Fisheries Research, 108, 336-343.

Alastair, V. H., Tobin, A. J., Simpfendorfer, C. A., Welch, D. J., Mapleston, A., White, J., Williams, A. J., \& Stapley, J. (2011). Evaluating catch and mitigating risk in a multispecies, tropical, inshore shark fishery within the Great Barrier Reef World Heritage Area. Marine and Freshwater Research, 62, 710-772.

Bessudo, S., Soler, G., Klimley, P., Ketchum, J., Hearn, A., \& Arauz, R. (2011). Residency of the scalloped hammerhead shark (Sphyrna lewini) at Malpelo Island and evidence of migration to other islands in the Eastern Tropical Pacific. Environmental Biology of Fishes, 91, 165-176.

Branstetter, S. (1987). Age and growth estimates for blacktip, Carcharhinus limbatus, and spinner, Carcharhinus brevipinna, sharks from the northwestern Gulf of Mexico. Copeia, 4, 964-974.

Bussing, W. A., \& López, M. I. (2005). Peces de la Isla del Coco y peces arrecifales de la costa Pacífica de América Central meridional/Fishes of Cocos Island and Reef Fishes of the Pacific Coast of Lower Central America. Revista de Biología Tropical, 53(Suplemento 2), 5-192.

Carr, L., Stier, A., Fietz, K., Montero, I., Gallagher, A., \& Bruno, J. (2013). Ilegal shark fishing in the Galápagos Marine Reserve. Marine Policy, 39, 317-321.

Castro, J. I. (1993). The nursery of Bull Bay, South Carolina, with a review of the shark nurseries of the Southeastern coast of the United States. Environmental Biology of Fishes, 38, 37-48.

Chiaramonte, G., \& Pettovello, A. (2000). The biology of Mustelus schmitti in southern Patagonia, Argentina. Journal of Fish Biology, 57, 930-942.

Cortés, J. (2008). Historias de las investigaciones marinas de la Isla del Coco, Costa Rica. Revista Biología Tropical, 56(Suplemento 2), 1-18.

Cortés, J., Sánchez-Jiménez, A., Rodríguez-Arrieta, J. A., Quirós-Barrantes, G., González, P. C., \& Blum, S. (2012). Elasmobranchs observed in deepwaters (45-330m) at Isla del Coco National Park, Costa Rica (Eastern Tropical Pacific). Revista de Biología Tropical, 60(Suplemento 3): 257-273.
Di Rienzo, J. A., Casanoves, F., Balzarini, M., Gonzalez, L., Tablada, M., \& Robledo, C. (2008). InfoStat, versión 2008. Grupo InfoStat, FCA, Universidad Nacional de Córdoba, Argentina.

Fitzpatrick, R., Abrantes, K., Seymour, J., \& Barnett, A. (2011). Variation in depth of whitetip reef sharks: does provisioning ecotourism change their behaviour? Coral Reefs, 30, 569-577.

Friedlander, A., Zgliczynski, B., Ballesteros, E., Aburto, O., Bolaños, A., \& Sala, E. (2012). The shallow-water fish assemblage of Isla del Coco National Park, Costa Rica: structure and patterns in an isolated, predatordominated ecosystem. Revista Biología Tropical 60(Suplemento 3), 321-338.

Gaitan, J., \& López, A. (2008). Presencia de juveniles de tiburón aletinegro Carcharhinus limbatus (Carcharhiniformes: Carcharhinidae) en la zona norte de la ecoregión Tayrona, Caribe colombiano. Latin American Journal of Aquatic Research, 36(1), 115-119.

Hanna, K., Foog, A., Driggers, W., Hoffmayer, E., Ingram, W., \& Grace, M. (2013). Size selectivity and catch rates of two small coastal shark species caught on circle. Fisheries Research, 147, 145-149.

Hearn, A., Klimley, P., \& Peñaherrera, C. (2010a). Hotspots within hotspots? Hammerhead shark movements around Wolf Island, Galapagos Marine Reserve. Marine Biology, 157, 1899-1915.

Hearn, A., Utreras, E., \& Henderson, S. (Eds.). (2010b). Informe sobre el estado de los tiburones del Pacífico Este Tropical, Vol. 1. Quito, Ecuador: Conservación Internacional.

Heupel, M., \& Simpfendorfer, C. (2008). Movements and distribution of young bull sharks (Carcharhinus leucas) in a variable estuarine environment. Aquatic Biology, 1, 277-289.

Heupel, M. R., Simpfendorfer, C. A., \& Hueter, R. E. (2004). Estimation of shark home ranges using passive monitoring techniques. Environmental Biology of Fishes, 71, 135-142.

Kitchell, J. F., Essington, T. E., Boggs, C. H., Schindler D. E., \& Walters, C. J. (2002). The role of sharks and Longline fisheries in a pelagic ecosystem of the Central Pacific. Ecosystems, 5, 202-216.

Klimley, A. P. (1987). The determinants of sexual segregation in the scalloped hammerhead shark, Sphyrna lewini. Environmental Biology of Fishes, 18, 27-40.

López, A., Zanella, I., Golfín, G., \& Pérez, M. (2012). First record of the blacktip reef shark Carcharhinus melanopterus (Carcharhiniformes: Carcharhinidae) from the Tropical Eastern Pacific. Revista Biología Tropical, 60(Suplemento 3), 275-278.

López, A., Zanella, I., Martínez, F., Golfín, G., \& Pérez, M. (2016). Caracterización de las especies pelágicas capturadas por las pesquerías ilegales en el Parque 
Nacional Isla del Coco, Costa Rica. Revista Biología Tropical, 64(Suplemento 1), 249-261.

McCauley, D. J., Mclean, K. A., Bauer, J., Young, H., \& Micheli, F. (2012). Evaluating the performance of methods for estimating the abundance of rapidly declining coastal shark populations. Ecological Applications, 22, 385-392.

McCord, M., \& Lamberth, S. (2009). Catching and tracking the world's largest Zambezi (bull) shark Carcharhinus leucas in the Breede Estuary, South Africa: the first 43 hours. African Journal of Ecology, 31, 107-111.

Merson, R. R., \& Pratt, H. L. (2001). Distribution, movements and growth of young sandbar sharks, Carcharhinus plumbeus, in the nursery grounds of Delaware Bay. Environmental Biology of Fishes, 61, 13-24.

Musick, J. A., Harbin, M. M., \& Compagno, L. J. V. (2004). Historical zoogeography of the Selachii. En C. Carrier, J. A. Musick \& M. R. Heithaus (Eds.). Biology of Sharks and Their Relatives (pp. 42-105). Boca Raton, Florida, Estados Unidos: CRC Press.

Myers, R. \& Worm, B. (2003). Rapid worldwide depletion of predatory fish communities. Nature, 423, 280-283.

Myers, R., Baum, J. K., Shepered, T. D., Powers, S., \& Peterson, C. H. (2007). Cascading effects of theloss of apex predatory sharks from a coastal ocean. Science, 315; 1846-1850.

Nalesso, E. (2014). Distribución espacio-temporal de los tiburones martillo, Sphyrna lewini, alrededor de la Isla del Coco (2005-2013), Pacífico Tropical Oriental. (Tesis de Maestría). Centro de Investigación Científica y de educación Superior de Ensenada, Baja California, Ensenada, Baja California, México.

Nelson, D. R., \& Johnson, R. H. (1980). Behaviour of the reef sharks of Rangiroa, French Polynesia. National Geographic Society Research Report, 12, 479-499.

Randall, J. (1977). Contribution to the biology of the whitetip reef shark (Triaenodon obesus). Pacific Science, 31, 143-164.

Robbins, W. (2006). Abundance, demography and population structure of the grey reef shark (Carcharhinus amblyrhynchos) and the whitetip reef shark (Triaenodon obesus) (Fam. Carcharhinidae). (Tesis Doctoral). James Cook University, Townsville, Australia.

Rowe, S. \& Hutchings, J. (2003). Mating system and the conservation of commercially exploited marine fishes. Trends in Ecology and Evolution, 18, 567-572.

Sibaja-Cordero, J. A., \& Cortés, J. (2010). Comparación temporal de la composición y zonación de organismos en el intermareal rocoso del Parque Nacional Isla del Coco, Pacífico de Costa Rica. Revista Biología Tropical, 58(Suplemento 4), 1387-1403.

Sibaja-Cordero, J. A., Troncoso, J., Benavides C., \& Cortés, J. (2012). Distribution of shallow water soft and hard bottom seabeds in the Isla del Coco National Park, Pacific Costa Rica. Revista Biología Tropical, 60(Suplemento 3), 53-66.

Simpfendorfer, C. A., \& Milward, N. E. (1993). Utilization of a tropical bay as a nursery area by sharks of the families Carcharhinidae and Sphyrnidae. Environmental Biology of Fishes, 37, 337-345.

Sinergia 69 (2002). Anexo V: Caracterización de las bahías de Chatham y Wafer e inventario preliminar de especies marinas. Área de Conservación Marina Isla del Coco (ACMIC). Proyecto: Estudio de factibilidad y diseño para la construcción de infraestructura y servicios de apoyo en el Parque Nacional Isla del Coco. San José, Costa Rica: Sinergia.

Tavares, R. (2005). Abundancia y distribución de tiburones en el Parque Nacional Archipiélago de Los Roques y otras islas oceánicas venezolanas, 1997-1998. Ciencias Marinas, 31, 441-454.

Tavares, R. (2008). Occurrence, diet and growth of juvenile Blacktip Sharks, Carcharhinus limbatus, from Los Roques Archipelago National Park, Venezuela. Caribbean Journal of Science, 44, 291-302.

Torres, H. A. M. (2004). Distribución, abundancia y hábitos alimentarios de juveniles del tiburón martillo Sphyrna lewini Griffith y Smith (Sphyrnidae) en la costa de Sinaloa, México durante el evento El Niño 1997-98. (Tesis de Maestría). Universidad del Mar, Oaxaca, México.

Walker, T. I. (1998). Can shark resources be harvested sustainably? A question revisited with a review of shark fisheries. Marine Freshwater Research, 49, 553-572.

Whitney, N., Papastamatiou, Y., Holland, K., \& Lowe, C. (2007). Use of an acceleration data logger to measure diel activity patterns in captive whitetip reef sharks, Triaenodon obesus. Aquatic Living Resources, 20, 299-305.

Zanella, I., López, A., \& Arauz, R. (2009). Caracterización de las descargas del tiburón martillo, Sphyrna lewini, en un área de crianza ubicada en el Golfo de Nicoya, Costa Rica. Revista de Ciencias Marinas y Costeras, 1, 175-195.

Zanella, I., López, A., Golfín, G. \& Sáenz, J. (2012). Abundancia, tamaño y estructura poblacional del tiburón punta blanca de arrecife, Triaenodon obesus (Carcharhiniformes: Carcharhinidae), en Bahía Chatham, Parque Nacional Isla del Coco, Costa Rica. Revista Biología Tropical, 60(Suplemento 3), 339-346. 